FERMILAB-Conf-02/236-E

\title{
First Observation of a Family of Double-Charm Baryons*
}

J. S. Russ ${ }^{\mathrm{a}}$

on behalf of the SELEX Collaboration ${ }^{\dagger}$

${ }^{a}$ Carnegie Mellon University

Pittsburgh, PA 15213 USA

The SELEX experiment (E781) at Fermilab has candidates for high mass states decaying to $\Lambda_{c}^{+} \mathrm{K}^{-} \pi^{+}$and $\Lambda_{c}^{+} \mathrm{K}^{-} \mathrm{pi}^{+} \pi^{+}$, Cabibbo-allowed decay modes of doubly-charmed baryons $\Xi_{c c}^{+}$and $\Xi_{c c}^{++}$. The masses are consistent with theoretical considerations, but the spectroscopy is surprising. Limited lifetime information suggests that $\tau_{\Xi_{c c}^{+}}^{++} \sim \tau_{\Xi_{c c}^{+}}$

\section{Introduction}

The existence of baryons with two and three charm quarks is expected from our present understanding of hadronic structure. In the doublecharm system one expects a $\mathrm{J}=1 / 2$ ground state iso-doublet, termed $\Xi_{c c}^{+,++}$in PDG notation [1]. Most predictions for the masses of the $\mathrm{J}=1 / 2$ states and the $\mathrm{J}=3 / 2$ hyperfine excitations expect the ground state near $3.6 \mathrm{GeV} / \mathrm{c}^{2}$ and a hyperfine split of $60 \mathrm{MeV} / \mathrm{c}^{2}[2]$.

The production mechanism for double-charm states is not clear. Perturbative pictures treat production as successive $c \bar{c}$ pair production with enough spatial overlap to form hadrons. Such cross sections are small compared to single-charm production.

\footnotetext{
* Talk at the 31st International Conference on High Energy Physics (ICHEP 2002), Amsterdam, July 24 - 31, 2002. To be published in the proceeding.

†SELEX Collaboration: Ball State University, Bogazici University, Carnegie Mellon University, Centro Brasileiro de Pesquisas Fisicas (Rio de Janeiro), Fermi National Accelerator Laboratory, Petersburg Nuclear Physics Institute, IHEP (Beijing), IHEP (Protvino), ITEP (Moscow), Moscow State University, Max-Planck-Institut für Kernphysik (Heidelberg), Tel Aviv University, Universidad Autónoma de San Luis Potosí, University of Bristol, Universidade Federal da Paraiba, University of Iowa, University of Michigan-Flint, University of Rome "La Sapienza", INFN, University of São Paulo, University of Trieste.
}

\section{Features of the Selex spectrometer}

The SELEX experiment at Fermilab is a 3stage magnetic spectrometer [3]. The negative $600 \mathrm{GeV} / \mathrm{c}$ Fermilab Hyperon Beam had about equal fluxes of $\pi^{-}$and $\Sigma^{-}$. The positive beam was $92 \%$ protons. For charm momenta in a range of $100-500 \mathrm{GeV} / \mathrm{c}$ mass resolution is constant and primary (secondary) vertex resolution is typically $270(560) \mu \mathrm{m}$. A RICH detector labelled all particles above $25 \mathrm{GeV} / \mathrm{c}$ [4]. Details of single-charm analyses involving $\Lambda_{c}^{+} \rightarrow p K^{-} \pi^{+}$reconstructions can be found in $[5,6]$. The double-charm search discussed here began with the sample of $1630 \Lambda_{c}^{+}$ events used in the lifetime analysis [5].

\section{Double-charm Analysis}

This topological search for double-charm baryons asks for a decay vertex lying between the primary vertex and the observed $\Lambda_{c}^{+}$decay vertex. A Cabibbo-allowed $\Xi_{c c}^{+}$decay can give a final-state $\Lambda_{c}^{+}$, a $\mathrm{K}^{-}$, and a $\pi^{+}$, shown schematically in Fig. 1.

We reconstruct total charge states $\mathrm{Q}=1$ (neutral 2-prong vertex) and $\mathrm{Q}=2$ (positive 3-prong vertex) in separate reconstructions. Few of the tracks from the intermediate vertex are RICHidentified. We call the negative track a kaon in the right-sign reconstruction. The same events 


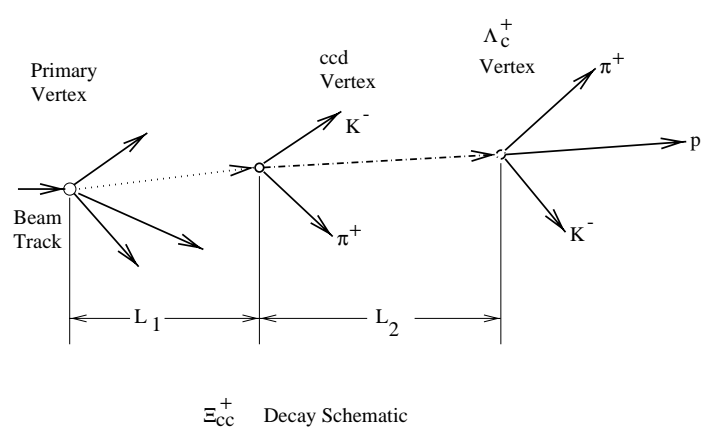

Figure 1. Schematic of $\Xi_{c c}^{+} \rightarrow \mathrm{K}^{-} \pi^{+} \Lambda_{\mathrm{c}}^{+}$

have a wrong-sign reconstruction, calling the negative track a pion.

Event selection cuts used here were taken without change from previous single-charm studies. For short-lived states, $\mathrm{L}_{1} / \sigma_{1} \geq 1$ and the $\Lambda_{c}^{+}$momentum vector must point back to the primary vertex within a $\chi^{2}$ cut of 4 . We have varied the cuts and observe that no signal significance depends critically on any cut value. The cuts were checked with simulation studies using several production models for double charm.

\section{1. $\mathrm{Q}=1$ reconstruction}

For single-charged baryons, the $\mathrm{K}^{-} \pi^{+} \Lambda_{\mathrm{c}}^{+}$mass distribution is shown in Fig. 2. Fig. 2(c) shows a $\Xi_{c c}^{+}$candidate at $3520 \mathrm{MeV} / \mathrm{c}^{2}$, consistent with most model calculations. The peak is narrow but consistent with simulation. The general agreement between right-sign (a,c) and wrong-sign (b) average levels and fluctuations in Fig. 2 confirms that most events are combinatoric background. The signal region in (c) contains 22 events with a background of $6.1 \pm 0.51$ events, for a singlebin significance of $6.3 \sigma$. The probability of such an excess is less than $10^{-6}$ for a single bin. We searched for a peak in the interval $3.2-4.3 \mathrm{GeV} / \mathrm{c}^{2}$, or 110 bins. The probability of such a fluctuation anywhere in the search interval is $<1.1 \times 10^{-4}$. SELEX has reported this as the first observation of a doubly-charmed baryon [7].

Using the reduced proper decay time $t^{*}=$ $M\left(L-L_{\min }\right) / p c$ we find that the lifetime $<33$

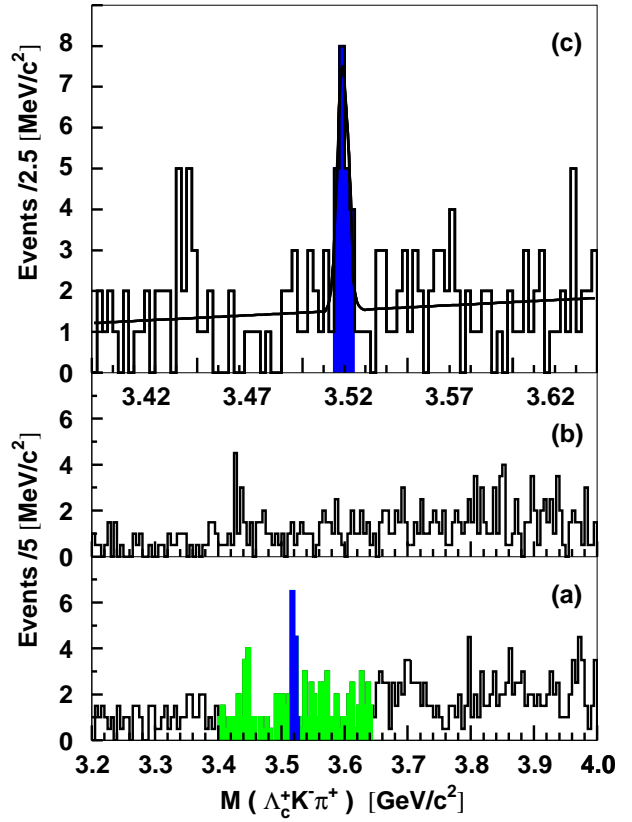

Figure 2. (a) The $\Lambda_{c}^{+} K^{-} \pi^{+}$mass distribution in $5 \mathrm{MeV} / c^{2}$ bins. The shaded region is shown in more detail in (c). (b) The wrong-sign combination $\Lambda_{c}^{+} K^{+} \pi^{-}$mass distribution in $5 \mathrm{MeV} / c^{2}$ bins. (c) The signal (shaded) region (22 events) and sideband mass regions (140 events) in 2.5 $\mathrm{MeV} / \mathrm{c}^{2}$ bins. The fit is a Gaussian plus linear background.

fs at $90 \%$ confidence. Here $\mathrm{L}_{\min }=\sigma_{1}$ the error on the vertex separation $\mathrm{L}_{1}$. The SELEX proper time resolution is about $20 \mathrm{fs}$. With constructive interference between the two c-quark decay amplitudes along with the $\mathrm{W}$-exchange amplitudes, this state could have a lifetime shorter than the $\Xi_{c}^{0}$.

\section{2. $\mathrm{Q}=2$ Reconstruction}

For double-charged baryons, we look for an isospin partner of the $\Xi_{c c}^{+}(3520)$. The $\mathrm{K}^{-} \pi^{+} \pi^{+} \Lambda_{\mathrm{c}}^{+}$mass distribution in the vicinity of $3520 \mathrm{MeV} / \mathrm{c}^{2}$ is shown in Fig. 3, along with the 
wrong-sign background.

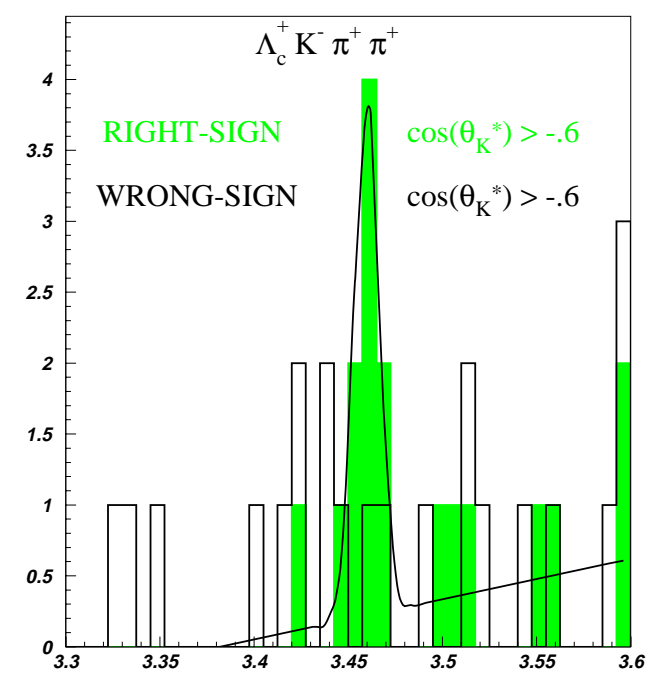

Figure 3. $\Xi_{c c}^{+} \rightarrow \mathrm{K}^{-} \pi^{+} \pi^{+} \Lambda_{\mathrm{c}}^{+}$mass distribution in $7.5 \mathrm{MeV} / \mathrm{c}^{2}$ bins. Signal events are shaded. Wrong-sign background is shown as open histogram boxes.

There is a $\Xi_{c c}^{++}$candidate at $3460 \mathrm{MeV} / \mathrm{c}^{2}$. The observed width $\left(6 \pm 1 \mathrm{MeV} / \mathrm{c}^{2}\right)$ matches simulation. Events outside the signal region show a strong preference for the center-of-mass (CM) angle of the negative track to be near 180 degrees. Simulation indicates that a cut to remove such events should have very little effect on the signal region for a phase-space decay distribution. That is indeed the case in the data. With the selection shown, we find 9 events in the peak, compared to an expected background of 1 event. The Poisson probability that there is an excess of 8 events or more anywhere on the plot is $10^{-5}$. There are too few events to attempt a lifetime analysis. The $\Xi_{c c}^{++}$candidates have a raw average proper time (uncorrected for acceptance) comparable to that for the $\Xi_{c c}^{+}$candidates, suggesting that $\tau_{\Xi_{c c}^{++}} \sim \tau_{\Xi_{c c}^{+}}$.

\subsection{Production}

Both $\Xi_{c c}^{+}$and $\Xi_{c c}^{++}$states are produced only by baryon beams in SELEX data. There are no signal candidates from the pion beam. Simulation studies suggest that the double-charm states may account for as much as $40 \%$ of the $\Lambda_{c}^{+}$sample seen in this experiment, a surprisingly high fraction. This situation is reminiscent of the discovery of the $\Xi_{c}^{+}$baryon in the WA62 experiment at CERN, using a $135 \mathrm{GeV}$ hyperon beam [8]. The FOCUS photoproduction experiment at Fermilab has looked for these states using their $\Lambda_{c}^{+}$events and sees no signal peaks [9]. If SELEX is correct, the hadroproduction mechanism is unusual.

\section{Summary}

SELEX has introduced two statisticallycompelling new high-mass states that decay into a final state $\Lambda_{c}^{+}, \mathrm{K}^{-}$and one or two $\pi^{+}$, as expected for double-charm baryon decays. The 3520 $\mathrm{MeV} / \mathrm{c}^{2}$ state satisifies all expectations for being a $\Xi_{c c}^{+}$state [7]. Its lifetime is shorter than $30 \mathrm{fs}$ at $90 \%$ confidence. The $3460 \mathrm{MeV} / \mathrm{c}^{2}$ state has the decay characteristics of a $\Xi_{c c}^{++}$state. It is difficult to understand the $60 \mathrm{MeV} / \mathrm{c}^{2}$ mass difference between the $Q=1$ and $Q=2$ states if they are members of the ground state isodoublet. However, any other interpretation is also problematic.

\section{REFERENCES}

1. Particle Data Group, D.E. Groom, et al., Eur. Phys. J.C15,1 (2000)

2. see references 111-124 in hep/ph0201071, B Physics at the Tevatron.

3. J. Russ, et al. , in Proceedings of the 29th International Conference on High Energy Physics, edited by A. Astbury et al. (World Scientific, Singapore, 1999) VolII, p. 1259;hep-ex/9812031.

4. J. Engelfried, et al., Nucl.Instr.and Methods A431, 53, 1999

5. A. Kushnirenko et al. Phys. Rev. Lett. 86, 5243 (2001), hep-ex/0010014.

6. F. Garcia et al. Phys. Lett. B528, 49 (2002), hep-ex/0109017. 
7. M. Mattson et al. Phys. Rev. Lett. 89, 112001 (2002)

8. S. Biagi, et al., Phys. Lett. B122, 455 (1983)

9. C. Riccardi, these proceedings. See also http://www.hep.vanderbilt.edu/ ${ }^{\sim}$ stenson/xicc/ xicc_focus.html 